PROCEEDINGS OF THE

AMERICAN MATHEMATICAL SOCIETY

Volume 126, Number 5, May 1998, Pages 1257-1265

S 0002-9939(98)04340-8

\title{
FREE PRODUCTS OF ABELIAN GROUPS IN THE UNIT GROUP OF INTEGRAL GROUP RINGS
}

\author{
ERIC JESPERS AND GUILHERME LEAL
}

(Communicated by Ronald M. Solomon)

\begin{abstract}
We classify finite groups $G$ which are such that the unit group of the integral group $\operatorname{ring} \mathbf{Z} G$ has a subgroup of finite index which is a non-trivial free product of abelian groups.
\end{abstract}

For a finite commutative group $G$ it is well known, and first discovered by Higman, that the unit group $\mathcal{U}(\mathbf{Z} G)$ of the integral group $\operatorname{ring} \mathbf{Z} G$ is the direct product of the trivial units and a finitely generated free abelian group. In particular, the structure of the unit group is known. For a nonabelian finite group $G$ the structure of the unit group $\mathcal{U}(\mathbf{Z} G)$ is much more complicated. The few known structure theorems state that $\mathcal{U}(\mathbf{Z} G)$ seldom belongs to a well studied class of groups, such as solvable and nilpotent groups (see for example $[9,10])$. The reason for this is that $\mathcal{U}(\mathbf{Z} G)$ contains a free subgroup of rank 2 except when $G$ is abelian or $G$ is a Hamiltonian 2-group, a result due to Hartley and Pickel. Recently, Marciniak and Sehgal explicitly described generators for such a free subgroup.

The study of free subgroups in $\mathcal{U}(\mathbf{Z} G)$ was continued in [4]; it is shown that there are only four finite groups $G$ which are such that $\mathcal{U}(\mathbf{Z} G)$ contains a noncyclic free subgroup of finite index. In [5] and [6] a classification is given of the finite groups $G$ which are such that $\mathcal{U}(\mathbf{Z} G)$ has a subgroup of finite index which is the direct product of noncyclic free subgroups.

In this paper we answer the following question posed to the authors by Z . Marciniak: describe the finite groups $G$ which are such that the unit group $\mathcal{U}(\mathbf{Z} G)$ contains a subgroup of finite index which is a free product of free abelian groups. As in $[4,5,6]$ it turns out that such groups can be characterised in terms of the Wedderburn decomposition of their rational group algebra. We will actually answer a slightly more general question.

Throughout the paper the following notation is used. The centraliser of an element $g$ of a group $G$ is denoted by $C_{G}(g)$. The dihedral group of order $2 n$ is denoted $D_{2 n}$, the quaternion group of order $2 n$ is denoted $Q_{2 n}$ and the cyclic group of order $n$ is denoted $C_{n}$. We also need some additional notation for four particular

Received by the editors October 7, 1996 .

1991 Mathematics Subject Classification. Primary 16U60, 16 S34.

The first named author is supported in part by NSERC grant OGP0036631, Canada.

The second named author, partially supported by CNPq, Brazil, wishes to thank the Memorial University of Newfoundland for its support and friendly atmosphere.

(C)1998 American Mathematical Society 
groups of order 16 and one of order 32 :

$$
\begin{aligned}
\mathcal{P}= & \left\langle a, b \mid a^{4}=1=b^{4}, b a=a^{3} b\right\rangle, \\
\mathcal{D}= & \left\langle a, b, c \mid a^{2}=b^{2}=c^{4}=1, a c=c a, b c=c b, b a=c^{2} a b\right\rangle, \\
D_{16}^{+}= & \left\langle a, b \mid a^{8}=b^{2}=1, b a=a^{5} b\right\rangle, \\
D_{8} Y Q_{8}= & \text { the central product of } D_{8} \text { and } Q_{8}, \\
& \text { with their respective centres amalgamated }
\end{aligned}
$$

By $M_{n}(R)$ we denote the $n$-by- $n$ matrix ring over a ring $R$, and by $\mathbf{H}^{t}(K)$ we denote a totally definite quaternion algebra over a real field $K$. By $\mathbf{H}(K)$ we denote the classical quaternion algebra over a field $K$. The unit group of a ring $R$ is denoted by $\mathcal{U}(R)$. For a subgroup $H$ of a finite group $G$ we denote by $\widehat{H}$ the idempotent $\frac{1}{|H|} \sum_{h \in H} h$ of $\mathbf{Q} G$.

We are now able to state the theorem.

Theorem 1. Let $G$ be a finite nonabelian group which is not a Hamiltonian 2group. Consider the following properties:

(1) $\mathcal{U}(\mathbf{Z} G)$ contains a subgroup of finite index which is a non-trivial free product of abelian groups.

(2) For any non-torsion unit $u \in \mathcal{U}(\mathbf{Z} G)$ there exists a positive integer $n$ such that $C_{\mathcal{U}(\mathbf{Z} G)}\left(u^{n}\right)$ is abelian-by-finite.

(3) The simple Wedderburn components of $\mathbf{Q} G$ are of one of the following six types: $\mathbf{Q}, K, \mathbf{H}^{t}(\mathbf{Q}), M_{2}(\mathbf{Q}), M_{2}(K)$ or $M_{2}\left(\mathbf{H}^{t}(\mathbf{Q})\right)$, where $K$ is a quadratic imaginary extension of $\mathbf{Q}$. Moreover, exactly one matrix component occurs.

(4) The group $G$ is isomorphic to one of the following: $D_{6}, D_{8}, Q_{12}, \mathcal{P}, \mathcal{D}, \mathcal{D}_{16}^{+}$, $Q_{8} \times C_{3}, Q_{8} \times C_{4}$ and $D_{8} Y Q_{8}$.

Then $(1) \Rightarrow(2) \Rightarrow(3) \Rightarrow(4)$.

Proof. $(\mathbf{1}) \Rightarrow(\mathbf{2})$. Let $H$ be a subgroup of finite index, say $n$, in $\mathcal{U}(\mathbf{Z} G)$ which is a free product of abelian groups $H_{1}, H_{2}, \cdots, H_{l}$. Then, for any non-torsion unit $u$ of $\mathbf{Z} G, 1 \neq u^{n} \in H$. The description of subgroups of free products, given by Kurosh (see for example [8, Theorem 6.3.1]), implies that $C_{H}\left(u^{n}\right)$ is a free product of a free group and conjugates of subgroups of the abelian groups $H_{i}, 1 \leq i \leq l$. Since the centre of a free product of two nontrivial groups is trivial (see for example [8, Proposition 6.2.6]) and because $1 \neq u^{n}$ is in the centre of $C_{H}\left(u^{n}\right)$, it follows that $C_{H}\left(u^{n}\right)$ is abelian. Because $H$ is of finite index in $\mathcal{U}$, it is then clear that $C_{H}\left(u^{n}\right)=C_{\mathcal{U}(\mathbf{z} G)}\left(u^{n}\right) \cap H$ is of finite index in $C_{\mathcal{U}(\mathbf{Z} G)}\left(u^{n}\right)$. So this shows that (2) holds.

$(\mathbf{2}) \Rightarrow(\mathbf{3})$. Write $\mathbf{Q} G=\bigoplus M_{n_{i}}\left(D_{i}\right)$, where $m_{i} \geq 1$ and each $D_{i}$ is a division algebra. Let $\mathcal{O}_{i}$ be a $\mathbf{Z}$-order in $D_{i}$; then both $\mathbf{Z} G$ and $\Lambda=\bigoplus M_{n_{i}}\left(\mathcal{O}_{i}\right)$ are $\mathbf{Z}$ orders in $\mathbf{Q} G$. Hence, there exists a positive integer $n(\Lambda)$ such that $n(\Lambda)$-th power of a unit of $\Lambda$ is a unit in $\mathbf{Z} G$. Hence, by [10, Proposition 1.9], periodic subgroups of $\mathcal{U}(\mathbf{Z} G)$ and of $\mathcal{U}\left(M_{n_{i}}\left(\mathcal{O}_{i}\right)\right)$ are of bounded period. Because such groups are also linear, it follows that periodic subgroups are finite.

If $e_{i}$ is the identity of $M_{n_{i}}\left(\mathcal{O}_{i}\right)$, we often identify $\mathcal{U}\left(M_{n_{i}}\left(\mathcal{O}_{i}\right)\right)$ with $\left(1-e_{i}\right)$ $+\mathcal{U}\left(M_{n_{i}}\left(\mathcal{O}_{i}\right)\right) \subseteq \mathbf{Q} G$. So, under this identification, $\mathbf{Z} G \cap\left(\left(1-e_{i}\right)+\mathcal{U}\left(M_{n_{i}}\left(\mathcal{O}_{i}\right)\right)\right)$ will be written as $\mathbf{Z} G \cap \mathcal{U}\left(M_{n_{i}}\left(\mathcal{O}_{i}\right)\right)$.

First we show that each $n_{i} \leq 2$. Assume the contrary; that is, suppose $m=n_{i} \geq$ 3. Let $E_{i j}, 1 \leq i, j \leq m$, be a set of matrix units for $M_{m}\left(\mathcal{O}_{m}\right)$. Then there exists a positive integer $n(\Lambda)$ such that the group $H=\left\langle 1+n(\Lambda) E_{i j} \mid m \geq j>i>1\right\rangle$ 
$\subseteq \mathcal{U}(\mathbf{Z} G)$. Since $H$ is not abelian and has a non-periodic central element, this yields a contradiction.

Second, we show that there exists precisely one index $i$ so that $M_{n_{i}}\left(\mathcal{O}_{i}\right)$ has infinitely many units, or equivalently the unit group of $M_{n_{i}}\left(\mathcal{O}_{i}\right)$ is non-periodic. By the Hartley-Pickel result and its proof (see Theorem 5.1 in [10]), and because $G$ is not abelian and not a Hamiltonian 2-group, there exists an index $j$ so that $\mathcal{U}\left(M_{n_{j}}\left(\mathcal{O}_{j}\right)\right) \cap \mathcal{U}(\mathbf{Z} G)$ contains a non-cyclic free group $F$. Suppose now that $i \neq j$ and $\mathcal{U}\left(M_{n_{i}}\left(\mathcal{O}_{i}\right)\right)$ is infinite. So let $u \in \mathcal{U}\left(M_{n_{i}}\left(\mathcal{O}_{i}\right)\right)$ be a non-periodic unit. Thus, there exists a positive integer $n(\Lambda)$ so that $1+u^{n(\Lambda)} \in \mathcal{U}\left(M_{n_{i}}\left(\mathcal{O}_{i}\right)\right) \cap \mathcal{U}(\mathbf{Z} G)$, and clearly $F \subset C_{\mathcal{U}(\mathbf{Z} G)}\left(u^{n(\Lambda)}\right)$, in contradiction with (2). This shows the claim.

Next we show that there exists a unique index $i$ with $n_{i} \neq 1$. Suppose the contrary, that is, $\mathbf{Q} G$ is a direct sum of division rings and fields. Hence $G$ is a Hamiltonian group, and since it is not a two group, $G$ contains $H=Q_{8} \times C_{p}$ with $p$ an odd prime. As $\mathbf{Q} H$ does not contain non-zero nilpotent elements, $p \geq 7$ (Lemma VI.1.18 in [9]). Hence the Wedderburn decomposition of $\mathbf{Q}\left(Q_{8} \times C_{p}\right)$ contains the direct sum $\mathbf{Q}\left(\zeta_{p}\right) \oplus \mathbf{H}\left(\mathbf{Q}\left(\zeta_{p}\right)\right)$.

Now $\mathcal{U}(\mathbf{Z} H) \cap \mathcal{U}\left(\mathbf{Z}\left[\zeta_{p}\right]\right)$ contains a non-periodic element $u$ and $\mathcal{U}(\mathbf{Z} H) \cap \mathcal{U}\left(\mathbf{H}\left[\mathbf{Z} \zeta_{p}\right]\right)$ contains a non-cyclic free group (Hartley- Pickel) $F_{2}$. Clearly $F_{2} \subset C_{\mathcal{U}(\mathbf{Z} G)}(u)$, again in contradiction with (3).

So far we have shown that there exists a unique index $j$ with $n_{j}=2$, and that all other $n_{i}$ are 1 . Furthermore it follows that for every $i \neq j, \mathcal{U}\left(\mathcal{O}_{i}\right)$ is finite. We now show that also $\mathcal{U}\left(\mathcal{O}_{j}\right)$ is finite. For this notice that for any $u \in \mathcal{U}\left(\mathcal{O}_{j}\right) \cap \mathcal{U}(\mathbf{Z} G)$ there exists an integer $m \geq 2$ such that

$$
\begin{aligned}
& u_{1}=\left[\begin{array}{ll}
1 & m \\
0 & 1
\end{array}\right], \\
& u_{2}=\left[\begin{array}{cc}
1 & 0 \\
m & 1
\end{array}\right]
\end{aligned}
$$

and

$$
v=\left[\begin{array}{cc}
u^{m} & 0 \\
0 & u^{m}
\end{array}\right]
$$

belong to $\mathcal{U}(\mathbf{Z} G)$; so the group $\left\langle u_{1}, u_{2}\right\rangle$ is free and $\left\langle u_{1}, u_{2}\right\rangle \subset C_{\mathcal{U}(\mathbf{Z} G)}(v)$. It follows from the assumption (2) that $v$, and thus $u$, are periodic. Hence $\mathcal{U}\left(\mathcal{O}_{i}\right)$ is periodic and thus finite.

The Dirichlet Unit Theorem and Lemma 21.3 in [10] now yield that $\mathcal{O}_{i}$ is either the ring of integers of a totally imaginary quadratic extension of $\mathbf{Q}$, or an order in a totally definite quaternion algebra over $\mathbf{Q}$. In particular, (3) follows.

(3) $\Rightarrow(4)$ A finite group whose rational group algebra satisfies condition (3) we call admissible. Assume $G$ is admissible. From the group tables of groups of small order (see for example [11]) one can easily deduce that if $|G| \leq 32$ then $G$ is one of the groups listed in (4). Hence it is sufficient to establish the following five steps.

(i) If $\mathbf{Q} G$ does not have a non-commutative division ring as a simple component, then $|G| \leq 32$.

(ii) If $\mathbf{Q} G$ has a non-commutative division ring as a simple component, then $Q_{8}$ or $Q_{12}$ is a homomorphic image of $G$.

(iii) If $Q_{12}$ is a homomorphic image of $G$, then $G=Q_{12}$.

(iv) If $Q_{8}$ is a homomorphic image of $G$ and $|G|>32$, then $G$ is a 2-group and either $G / \mathcal{Z}(G) \cong C_{2} \times C_{2}$ or $G$ contains a Hamiltonian subgroup of index 2 . 
(v) If $G$ is a 2-group and $Q_{8}$ is a homomorphic image of $G$, then $|G| \leq 32$.

To prove these statements we need six technical lemmas.

Lemma 2. If $N$ is a subgroup of $G$, then $\mathbf{Q} N$ does not have two matrix rings as simple components, nor does it contain a matrix ring over a division ring with infinitely many units in its orders.

Proof. Since orders in matrix rings have infinitely many units, any matrix component of $\mathbf{Q} N$ is contained in the matrix component of $\mathbf{Q} G$. If $\mathbf{Q} N$ had two matrix components, then this would yield four orthogonal idempotents in a 2-by-2 matrix ring component of $\mathbf{Q} G$, which is impossible.

On the other hand if $M_{2}\left(D_{1}\right), D_{1}$ a division ring, is a simple component of $\mathbf{Q} H$, then it follows that $M_{2}\left(D_{1}\right) \subseteq M_{2}(D)=(\mathbf{Q} G) e$, where $e$ is the primitive central idempotent defining the matrix component of $\mathbf{Q} G$. Since, by assumption, orders in $D$ have a finite unit group, the same holds for orders in $D_{1}$.

Lemma 3. The possible orders of elements of $G$ are 1, 2, 3, 4, 6, 8 or 12, and the centre of $G$ has exponent at most 6 .

Proof. Let $g \in G$ be an element of order $n$. Consider the group algebra $\mathbf{Q}\langle g\rangle$ $=\mathbf{Q}\left(\zeta_{n}\right) \oplus M$ with $\mathbf{Q}\left(\zeta_{n}\right)=\mathbf{Q}\langle g\rangle f, \zeta_{n}$ a primitive $n$-th root of unity, $f$ a primitive idempotent and $M$ a direct sum of fields. Let $e$ be a primitive central idempotent of $\mathbf{Q} G$ such that $f e \neq 0$. So $\mathbf{Q}\left(\zeta_{n}\right) \subseteq(\mathbf{Q} G) e$.

From the assumptions we obtain that

$$
[(\mathbf{Q} G) e: \mathbf{Q}]=[(\mathbf{Q} G) e: \mathcal{Z}((\mathbf{Q} G) e)][\mathcal{Z}((\mathbf{Q} G) e): \mathbf{Q}]=2^{i},
$$

with $0 \leq i \leq 4$, and therefore $\varphi(n)=2^{i}, 0 \leq i \leq 4$. Note that actually $i<4$, for otherwise $(\overline{\mathbf{Q}}\langle g\rangle) e=(\mathbf{Q} G) e$ is a field of $\mathbf{Q}$-dimension 16, a contradiction. Clearly we obtain that $n=1,2,3,4,5,6,8,10,12,15,16,20,24$ or 30 .

In the cases $n=10,15,16,20,24$ or 30 , one can write

$$
\mathbf{Q}\langle g\rangle=\mathbf{Q}\langle g\rangle f_{1} \oplus \mathbf{Q}\langle g\rangle f_{2} \oplus M^{\prime},
$$

where $M^{\prime}$ is a direct sum of fields, and $F_{1}=\mathbf{Q}\langle g\rangle f_{1}$ and $F_{2}=\mathbf{Q}\langle g\rangle f_{2}$ are fields each of which have ring of integers with infinite group of units. Since orders in the division ring components of $\mathbf{Q} G$ have finite unit groups, it follows that $f_{1} e \neq 0$, $f_{2} e \neq 0$ and $\mathbf{Q} G e=M_{2}(D), D$ a division ring. Hence $F_{i} \subseteq e f_{i} \mathbf{Q} G e f_{i} \cong D$. Thus $D=F_{i}$, and thus an order in $D$ contains infinitely many units, again a contradiction. So we have shown that the order of $g$ is $1,2,3,4,5,6,8$ or 12 . From the above method it also follows that the centre of $G$ has exponent at most 6 .

So it remains to show that $n \neq 5$. Assume the contrary. Then $\mathbf{Q}\langle g\rangle=\mathbf{Q}\langle g\rangle \widehat{g} \oplus$ $\langle g\rangle \mathbf{Q}(1-\widehat{g}), \mathbf{Q}\langle g\rangle \widehat{g} \cong \mathbf{Q}$ and $\mathbf{Q}\langle g\rangle(1-\widehat{g}) \cong \mathbf{Q}\left(\zeta_{5}\right)$. Since the unit group of $\mathbf{Z}\left[\zeta_{5}\right]$ is infinite and because $G$ is admissible, $(1-\widehat{g}) e \neq 0,(\mathbf{Q} G) e$ is a matrix ring and $(1-\widehat{g}) f=0$ for any primitive central idempotent $f \in \mathbf{Q} G$ with $f \neq e$. Hence $1-\widehat{g}=(1-\widehat{g}) e$. Therefore the idempotent $1-\widehat{g}$ belongs to QGe. Now, $1-\widehat{g}$ is central, for otherwise $\mathbf{Q} \widehat{g}(1-\widehat{g}) \subseteq(1-\widehat{g}) e \mathbf{Q} G(1-\widehat{g}) \cong D$. So $D \cong \mathbf{Q}\left(\zeta_{5}\right)$ and therefore an order in $D$ has infinitely many units, a contradiction. Hence it follows that $1-\widehat{g}=e$. Since $e$ is central this implies that the group $\langle g\rangle$ is normal in $G$. Now, since $(\mathbf{Q} G) e$ is nonabelian, there exists $h \in G$ such that $h e \notin\langle g\rangle e$. Because $g$ has order 5 and the order of $h$ is $2,3,4,5,6,8$ or 12 , we may assume, by replacing $h$ by some power if necessary, that he has order 2, 3 or 5 . Furthermore, there must exist such an $h$ with order of he different from 5; for otherwise Ge is a 5-group and thus 
Q $G$ would not have a simple component which is a 2-by-2 matrix ring. If he has order 2 , then, since $G$ does not have elements of order $10,\langle g e, h e\rangle \cong D_{10}$. However, because of Lemma 2, this is impossible as $\mathbf{Q} D_{10}$ has $M_{2}(F)$ as a simple component, where $F$ a quadratic real field extension of $\mathbf{Q}$. If he has order 3 , then $\langle g e, h e\rangle$ is a group of order 15, and thus commutative. Again this yields a contradiction, as $G$ does not have elements of order 15 .

Lemma 4. If $N$ is a subgroup of $G$ and $\mathbf{Q} N$ has a simple component which is a matrix ring (that is, $N$ is nonabelian and not Hamiltonian), then $N$ is admissible. Furthermore, if $\mathbf{Q} N$ has a matrix component of the same $\mathbf{Q}$-dimension as the matrix component of $\mathbf{Q} G$, then $N=G$.

Proof. Let $f$ be a primitive central idempotent of $\mathbf{Q} N$ and let $e$ be a primitive central idempotent of $\mathbf{Q} G$ with ef $\neq 0$. Since $(\mathbf{Q} N) f$ is simple, $(\mathbf{Q} N) f \subseteq(\mathbf{Q} G) e$.

We first deal with the case that $(\mathbf{Q} N) f$ is a matrix ring. Note that, since $\mathbf{Q} G$ has only one matrix component, $e$ is the only primitive central idempotent of $\mathbf{Q} G$ with $e f \neq 0$. Hence $e f=f$. First assume $(\mathbf{Q} N) f e=(\mathbf{Q} G) e=M_{2}(D)$, where $D$ is a division ring (this occurs precisely when $\operatorname{dim}_{\mathbf{Q}}((\mathbf{Q} N) f e)=\operatorname{dim}_{\mathbf{Q}}((\mathbf{Q} G) e)$ ). Then $f=f e=e$. Hence $G f=G e \subseteq \mathbf{Q} N f$. Consequently, $G \subseteq \operatorname{supp}(G f) \subseteq$ $\operatorname{supp}((\mathbf{Q} N) f) \subseteq N$; and thus $G=N$.

Next assume $(\mathbf{Q} N) f$ is properly contained in $(\mathbf{Q} G) e$ and $(\mathbf{Q} N) f$ is a matrix ring. Then $\operatorname{dim}_{\mathbf{Q}}((\mathbf{Q} N) f)=4$ or 8 . So either $(\mathbf{Q} N) f=M_{2}(\mathbf{Q})$ or $(\mathbf{Q} N) f=M_{2}(F)$, where $F$ is a quadratic field extension of $\mathbf{Q}$. Now, if $(\mathbf{Q} N) f=M_{2}(F)$, then a set of matrix units of $(\mathbf{Q} N) f$ is also a set of matrix units of $(\mathbf{Q} G) e$; and thus the centraliser of these matrix units in $(\mathbf{Q} N) f$ is contained its centraliser in $(\mathbf{Q} G) e$. Thus $F \subset D$, where $D$ is a division ring such that $(\mathbf{Q} G) e=M_{2}(D)$. So the ring of integers $\mathcal{O}$ of $F$ is contained in an order of $D$. The latter has finitely many units; therefore the same holds for $\mathcal{O}$. Hence $F$ is quadratic imaginary. So the matrix component of $\mathbf{Q} N$ is of a good type.

Next assume $(\mathbf{Q} N) f=D$, a division ring. Note that the simple matrix component of $\mathbf{Q} N$ and $D$ cannot belong to the 2-by-2 matrix component of $\mathbf{Q} G$. So, up to isomorphism, $(\mathbf{Q} N) f=D$ is contained in a division ring component of $\mathbf{Q} G$. Since an order in the latter has finitely many units, the same holds for an order in $D$. Hence $\mathbf{Q} N f=\mathbf{Q} G e$ is a totally definite quaternion algebra over $\mathbf{Q}$; that is, it is again of a good type. It follows that $N$ is admissible.

Note that the rational group algebra of the Hamiltonian group $Q_{8} \times C_{3}$ has a matrix ring as simple component. It follows from the two previous lemmas that the only Hamiltonian subgroups or homomorphic images of $G$ which are such that their rational group algebra is a sum of division rings are Hamiltonian 2-groups. We will use this remark several times.

Lemma 5. If $N$ is a subgroup of $G$ such that $\mathbf{Q} N$ has a matrix ring and a noncommutative division ring as simple components, then $N=G$.

Proof. Let $e_{1}$ and $e_{2}$ be central primitive idempotents of $\mathbf{Q} N$ such that $\mathbf{Q} N e_{1}$ is a matrix ring and $\mathbf{Q} N e_{2}$ is a non-commutative division ring. Once again, it is clear that $(\mathbf{Q} N) e_{1}$ and $(\mathbf{Q} N) e_{2}$ are not contained in the same matrix simple component of $\mathbf{Q} G$. Since noncommutative division ring components of $\mathbf{Q} G$ have $\mathbf{Q}$-dimension $4, \mathbf{Q} N e_{2}=\mathbf{Q} G e$ is a division ring, for some primitive central idempotent $e$ of $\mathbf{Q} G$. Hence $G \subseteq \operatorname{supp}\left(G e_{2}\right) \subset \mathbf{Q} N e_{2} \subseteq N$, and thus $G=N$. 
Lemma 6. Assume $N$ is a nonabelian subgroup of $G$ which is not a Hamiltonian 2-group. If $|N| \geq 32$, then $N=G$.

Proof. Since $N$ is nonabelian, $\operatorname{dim}_{\mathbf{Q}} \mathbf{Q} N\left(1-\widehat{N^{\prime}}\right) \geq|N| / 2 \geq 16$. We consider two cases. First assume $\mathbf{Q} N\left(1-\widehat{N^{\prime}}\right)$ is simple. In particular, $\mathbf{Q} N$ has only one noncommutative simple component. If this component were a division ring, then $N$ would be a Hamiltonian 2-group, in contradiction with the assumption. So, by Lemma 4 , $\mathbf{Q} N\left(1-\widehat{N^{\prime}}\right)=M_{2}(D), D$ a division ring of dimension at most 4 over $\mathbf{Q}$. Because $\operatorname{dim}_{\mathbf{Q}} \mathbf{Q} N(1-\widehat{N}) \geq 16$, we obtain $\operatorname{dim}_{\mathbf{Q}} D=4$. Hence, by Lemma $4, N=G$.

Second, assume $\mathbf{Q} N\left(1-\hat{N}^{\prime}\right)$ is not simple; then it must contain a matrix ring and a non-commutative division ring. So, by Lemma $5, N=G$.

Lemma 7. If $N$ is a proper subgroup of $G$, then $N$ is abelian or a Hamiltonian 2-group, or $|N| \leq 16$.

Proof. By Lemma 5, $\mathbf{Q} N$ does not contain a matrix ring and a non-commutative division ring as simple components. So either $\mathbf{Q} N$ is a sum of division rings, and then $N$ is abelian or a Hamiltonian 2-group; or $\mathbf{Q} N$ has only one non-commutative component and this component is a matrix ring. By Lemma 4, this matrix ring is of the type $M_{2}(F)$ with $\mathbf{Q}$-dimension of $F$ equal to 1 or 2 . Then

$$
8 \geq \operatorname{dim}_{\mathbf{Q}} \mathbf{Q} N\left(1-\hat{N}^{\prime}\right) \geq N / 2 \text {. }
$$

So $|N| \leq 16$.

We now proceed with the proofs of the five earlier mentioned main steps.

Proof of (i). Suppose $G$ does not have a noncommutative division ring as simple component. Since $G$ is admissible, this means that $\mathbf{Q} G(1-\widehat{G})$ is a matrix ring. Hence $\operatorname{dim}_{\mathbf{Q}} \mathbf{Q} G(1-\widehat{G}) \leq 16$. Now, once again, $\mathbf{Q} G \cong \mathbf{Q}\left[G / G^{\prime}\right] \oplus \mathbf{Q} G\left(1-\widehat{G^{\prime}}\right)$. So it follows that $|G| \leq \frac{|G|}{2}+16$. Hence $|G| \leq 32$.

Proof of (ii). Assume $(\mathbf{Q} G) e$ is a noncommutative division ring component of $\mathbf{Q} G$, where $e$ is a primitive central idempotent. Because of Lemma 3, the only possible primes dividing $|G|$ are 2 and 3 , and $G$ does not contain elements of order 16 nor of order 9. Hence, since $G e$ is a subgroup of a division ring (and thus is fixed point free), it follows from [7, Theorem 18.1] that the 3-Sylow subgroups of Ge are cyclic, and thus of order 3 , and the 2-Sylow subgroups are either cyclic or a generalised quaternion group. Hence they are either $C_{2}, C_{4}, C_{8}, Q_{8}$ or $Q_{16}$. However, because of Lemma 4 , the group $Q_{16}$ is excluded. Hence $|G e|$ is 24,12 or 8 (note that $G e$ is nonabelian and cannot be of order 6). Since the group algebra $\mathbf{Q}[G e]$ has at most one matrix ring as a simple component, it follows from the Wedderburn decompositions of the groups of such orders that: if $|G e|=8$, then $G e=Q_{8}$; if $|G e|=12$, then $G e=Q_{12}$; if $|G e|=24$, then $G e=Q_{8} \times C_{3}$. However, $\mathbf{H}(\mathbf{Q})$ is the only noncommutative division ring simple component of the rational group algebra of the latter group. Since $Q_{8} \times C_{3}$ is not embedded in $\mathbf{H}(\mathbf{Q})$, this situation actually does not occur.

Proof of (iii). Let $N$ be a normal subgroup of $G$ with $G / N \cong Q_{12}$. Clearly $G$ contains normal subgroups $H_{1}$ and $H_{2}$ so that $N \subseteq H_{1} \subseteq H_{2}, G / H_{1} \cong D_{6}$ and $H_{2} / H_{1}$ is of order 3. By Lemma $3, G$ does not contain elements of order 9; hence there exists an element $g \in G$ of order 3 such that $H_{2} / H_{1}$ is generated by the natural image of $g$ in this group. 
We claim that $\langle g\rangle$ is a normal subgroup of $G$. Indeed, note that the subgroup $\left\langle g, H_{1}\right\rangle$ is normal in $G$ and the idempotent $\widehat{g}\left(1-\widehat{H_{1}}\right)$ belongs to $(\mathbf{Q} G)\left(1-\widehat{H_{1}}\right)$, a direct sum of division rings (note that $\mathbf{Q} G \widehat{H_{1}}$ contains a matrix ring as simple component). Hence, $\widehat{g} \widehat{H_{1}}$ and $\widehat{g}\left(1-\widehat{H_{1}}\right)$ are central in $\mathbf{Q} G$. Hence, $\widehat{g}$ is central and thus $\langle g\rangle$ is normal in $G$.

Again by Lemma 3, there exists $h \in G$ such that the order of $h$ is $2^{i}, 1 \leq i \leq 3$, and the natural image of $h$ in $G / H_{1}$ is of order 2. Then, since $\langle g\rangle$ is normal in $G, T=\langle g, h\rangle=\left\{h^{i} g^{j}\right\}$; and similarly as above we obtain that $\widehat{T}=\widehat{g} \widehat{h}$ is central in $\mathbf{Q} G$. Therefore $T$ is a normal nonabelian subgroup of $G$ with $|T|=2^{i} 3$. Thus $|T|=24,12$ or 6 .

If $|T|=6$ then $T \cong D_{6}$. It follows that $G \cong T \times H_{1} \cong D_{6} \times H_{1}$. Hence $\mathbf{Q} G$ has more than one simple component which is a matrix ring, a contradiction.

If $|T|=12$, then since $D_{6}$ is a homomorphic image of $T$, the subgroup $T$ is admissible. Hence $T=Q_{12}$, and thus, by Lemma $5, T=G$.

If $|T|=24$, once again as before one shows that $T$ is admissible. Thus $T=$ $C_{3} \times Q_{8}$, and thus it follows again that $T=G$. However, this is impossible, as $Q_{12}$ is not a homomorphic image of $C_{3} \times Q_{8}$.

So we have shown that $G=Q_{12}$.

Proof of (iv). Assume $G / N \cong Q_{8}$ and $|G|>32$. Because of Lemma 7, any subgroup containing $N$ and of index 2 in $G$ is either abelian or a Hamiltonian 2-group. In the latter case $G$ itself is a 2-group and we are done. So we may assume that all such subgroups are abelian. Then it follows easily that $G / \mathcal{Z}(G) \cong C_{2} \times C_{2}$. In particular $G$ is nilpotent, and thus $G$ is the direct product of its Sylow 2 and 3 subgroups, which we denote by $G_{2}$ and $G_{3}$ respectively. Because of Lemma $3, G_{3} \subseteq N$ is an elementary abelian 3 -group. Note that $G_{2}$ is nonabelian. If $\mathbf{Q} G_{2}$ contains a matrix ring as a simple component, then clearly $G_{3}$ is trivial, as $G$ is admissible. So $G_{2}$ is a Hamiltonian 2-group. We claim that $G_{3}$ is trivial. For otherwise, $Q_{8} \times C_{3}$ is a subgroup of $G$. Since $\mathbf{Q}\left[Q_{8} \times C_{3}\right]$ contains a matrix ring and a noncommutative division ring as simple components, it follows from Lemma 5 that $G=Q_{8} \times C_{3}$, a contradiction as $|G|>32$.

Proof of (v). Assume $G$ is a 2-group with normal subgroup $N$ such that $G / N=Q_{8}$. We have to prove that $|G| \leq 32$. Suppose the contrary. By step (iv), either $G / \mathcal{Z}(G) \cong C_{2} \times C_{2}$ or $G$ contains a Hamiltonian normal subgroup $N$ of index 2 .

First we deal with the case that $G / \mathcal{Z}(G) \cong C_{2} \times C_{2}$. Note that then $G^{\prime}=$ $\{1, c\}$, with $c$ a central element of order 2 , and that $\mathcal{Z}(G)$ has exponent 2 or 4 (by Lemma 3$)$. So $(\mathbf{Q} G) \frac{1-c}{2}$ is the direct sum of all the noncommutative simple components of $\mathbf{Q} G$, and all these simple components are generalised quaternion algebras over $\mathbf{Q}$ or $\mathbf{Q}(i)$. In the latter case such a simple component is a matrix ring. Hence, since $G$ is admissible, there exists at most one matrix component over $\mathbf{Q}(i)$. We claim that $\mathcal{Z}(G)$ is an elementary abelian 2-group. Suppose the contrary. Since $|G|>32,|\mathcal{Z}(G)|>8$ and thus $\mathcal{Z}(G)$ contains $C_{4} \times C_{2}$. It follows that $\mathbf{Q}\left[C_{4} \times C_{2}\right] \frac{1-c}{2}$ is contained in the centre of $(\mathbf{Q} G) \frac{1-c}{2}$ and contains 2 copies of the field $\mathbf{Q}(i)$, a contradiction. So indeed $\mathcal{Z}(G)$ has exponent 2, and therefore there exist elements $a, b \in G$ such that both $a$ and $b$ have order 4 and $Q_{8}$ is an epimorphic image of $\langle a, b\rangle$. Since $c \in\langle a, b\rangle$ and because $c$ has order 2, the group $\langle a, b\rangle$ is normal in $G$ and is of order at most 32. Because $\mathcal{Z}(G)$ is of exponent 2, it follows that $G \cong\langle a, b\rangle \times E$, where $E$ is an elementary abelian 2-group. As $G$ is admissible, the 
group algebra $\mathbf{Q}\langle a, b\rangle$ contains a matrix ring as a simple component. Furthermore, because $\mathbf{Q} G$ has precisely one matrix ring as simple component, we obtain that $E$ is trivial. This finishes the proof for this type of groups.

Next assume $N$ is a Hamiltonian subgroup of index 2 in $G$. Therefore $Q_{8}=$ $\langle i, j\rangle \subseteq N$ and $N=Q_{8} \times E$, with $E$ an elementary abelian 2-group. Note that $i^{2}$ is central in $G$ as $N$ is normal in $G$ and $i^{2}$ is the unique nontrivial commutator in $N$. Let $a \in G$ be such that its natural image in $G / N$ generates this group. We claim that we may assume that $Q_{8}$ is normal in $G$. This is clear if the cyclic groups $\langle i\rangle$ and $\langle j\rangle$ are normal in $G$. On the other hand, if, say, $\langle i\rangle$ is not normal, then take for $Q_{8}$ the group $\left\langle i, a i a^{-1}\right\rangle$. Next we claim that $E$ is not central. For otherwise, one can write $G=\left\langle Q_{8}, a\right\rangle \times T$, for some subgroup $T$ of $E$. Since $G$ is admissible, this implies that $T$ is trivial, and thus $|G| \leq 32$, a contradiction.

Let $c_{1} \in E$ be non-central in $G$. Because $\frac{1-i^{2}}{2}$ is a central idempotent, one of the idempotents $\left(\frac{1-i^{2}}{2}\right)\left(\frac{1-c_{1}}{2}\right)$ or $f=\left(\frac{1+i^{2}}{2}\right)\left(\frac{1-c_{1}}{2}\right)$ is central. It is easily seen that the former one does not commute with $a$. Hence $f=a f a^{-1}=\left(\frac{1+i^{2}}{2}\right)\left(\frac{1-c_{2}}{2}\right)$, where $c_{2}=a c_{1} a^{-1} \neq c_{1}$. Consequently, $c_{1}=i^{2} c_{2}$.

Write $a^{2}=q e$ with $q \in Q_{8}$ and $e \in E$. Then, $q e=a a^{2} a^{-1}=a q e a^{-1} \in Q_{8} a e a^{-1}$. So, $e\left(a e a^{-1}\right)$ is an element of order at most 2 in $Q_{8}$, and thus either $e a=e a$ (that is, $e$ is central in $G$ ) or $a e a^{-1}=i^{2} e$.

Assume $e$ is central. Then $f_{2}=\frac{1+e}{2}$ and $f_{3}=\frac{1-e}{2}$ are orthogonal central idempotents. So one of the idempotents $f_{2} \frac{1+c_{1}}{2}, f_{3} \frac{1+c_{1}}{2}$ has to be central. Because $a c_{1} a^{-1}=i^{2} c_{1}$, this is only possible if $e=1$. But then the group $\left\langle Q_{8}, a, c_{1}\right\rangle$ has order 32. Since its group algebra has non-central idempotents, this group is admissible (by Lemma 4). Since not all its elements of order 2 are central, this group is not Hamiltonian. Hence it must be the group $D_{8} Y Q_{8}$. Lemma 4 then yields that $G=D_{8} Y Q_{8}$.

Next assume $a e a^{-1}=i^{2} e$. Then the group algebra of the group $H=\left\langle Q_{8}, a, e\right\rangle$ has $\frac{1+e}{2}$ as a non-central idempotent. So $\mathbf{Q} H$ contains a matrix component, and thus, by Lemma $4, H$ is admissible. Since $|H|=32$ and because $H$ has noncentral elements of order 2, we obtain that $H=D_{8} Y Q_{8}$. Hence, by Lemma $4, H=G$.

As mentioned earlier, from the group tables of groups of small order one obtains that $(4) \Rightarrow(3)$. Since the groups listed in (4) only have one simple component with infinitely many units, and because the centraliser of a non-central element of $G L_{2}(\mathbf{C})$ is abelian, one obtains that $(4) \Rightarrow(2)$, except possibly for $D_{8} Y Q_{8}$.

Finally we remark that finite groups with reduced Q-degrees either 1 or a fixed number $k$ have been investigated in $[2,3]$. Among the several results obtained, it is shown that such groups are metabelian. If furthermore these groups have a faithful irreducible representation, then a classification of these groups can be deduced from the methods used in $[2,3]$. Note that subgroups of $M_{2}(D), D$ a division ring, also have been classified by Banieqbal in [1]. The use of these papers could potentially simplify some of our proofs. However, we elected to present a comprehensive proof.

\section{REFERENCES}

[1] B. Banieqbal, Classification of finite subgroups of $2 \times 2$ matrices over a division algebra of characteristic zero, J. Algebra 119 (1988), 449-512. MR 90h:20073

[2] R. Gow and B. Huppert, Degree problems of representation theory over arbitrary fields of characteristic 0, J. reine angew. Math. 381 (1987), 136-147. MR 89b:20029 
[3] R. Gow and B. Huppert, Degree problems of representation theory over arbitrary fields of characteristic 0, Part 2: Groups which have only two reduced degrees, J. reine angew. Math. 389 (1988), 122-132. MR 89i:20021

[4] E. Jespers, Free normal complements and the unit group of integral group rings, Proc. Amer. Math. Soc. 122(1) (1994), 59-66. MR 94k:16058

[5] E. Jespers, G. Leal and A. del Rio, Products of free groups in the unit group of integral group rings, J. Algebra 180 (1996), 22-40. MR 96m:16045

[6] G. Leal and A. del Rio, Products of free groups in the unit group of integral group rings II, J. Algebra 191 (1997), 240-251. CMP 97:11

[7] D.S. Passman, Permutation groups, Benjamin, New York, 1968. MR 38:5908

[8] D.J.S. Robinson, A course in the theory of groups, Graduate Texts in Math. 80, SpringerVerlag, New York, 1982. MR 84k:20001

[9] S.K. Sehgal, Topics in group rings, Marcel Dekker, New York, 1978. MR 80j:16001

[10] S.K. Sehgal, Units of Integral Group Rings, Longman Scientific and Technical, Essex, 1993. MR 94m: 16039

[11] A.D. Thomas and G.V. Wood, Group Tables, Shiva Publishing Limited, 1980. MR 81d:20002

Department of Mathematics and Statistics, Memorial University of Newfoundland, St. John's, Newfoundland, CAnada A1C 5S7

E-mail address: ejespers@albert.math.mun.ca

Instituto de Matemática, Universidade Federal do Rio de Janeiro, Rio de Janeiro RJ, BRAZIL

E-mail address: gleal@mat.dme.ufrj.br 